\title{
A Multispectral 3D-Endoscope for Cholesteatoma Removal
}

\author{
Eric L. Wisotzky ${ }^{1,2,}$, Jean-Claude Rosenthal ${ }^{1,}$, Anna Hilsmann ${ }^{1}$, Peter Eisert ${ }^{1,2}$ and Florian C. Uecker ${ }^{3}$ \\ ${ }^{1}$ Fraunhofer HHI, Vision and Imaging Technologies, Berlin, Germany \\ ${ }^{2}$ Humboldt Universiät zu Berlin, Visual Computing, Berlin, Germany \\ ${ }^{3}$ Charité - Universitätsmedizin Berlin, Department of Otorhinolaryngology, Berlin, Germany \\ $\$$ These authors contributed equally to this work.
}

\section{Introduction}

Medical imaging systems like surgical microscopes or endoscopes are indispensable in the daily work for surgeons already for a long time. Nowadays, these systems have evolved to a complete new level by offering fully digitized processing chains. Especially, image-based surgical guidance can benefit from these technical advancements, mainly for highly challenging surgeries like cholesteatoma removal.

Cholesteatoma is a destructive proliferating tissue. It can lead to deafness when the ossicular chain is damaged or the cochlea is breached. The only treatment for this disease is surgery. During surgery, it is very difficult to differentiate between healthy tissues and cholesteatoma. Moreover, it can even recur if not all tissue particles of cholesteatoma are removed, resulting in unwanted consecutive surgery.

\section{Methods}

We developed a stereo-multispectral endoscopic prototype using a filter-wheel for surgical guidance to remove cholesteatoma tissue in the middle ear. This image-based method combines multispectral tissue classification for cholesteatoma identification, 3D-reconstruction to detect all removal parts and determine its metric dimensions intraoperatively. The multispectral illumination ranges from $\lambda=400 \mathrm{~nm}$ to $\lambda=500 \mathrm{~nm}$ with step-sizeo of $20 \mathrm{~nm}$, which results in six different narrow-band illumination modes. For classical RGB imaging, a broadband illumination mode is applied before and after the narrow-band illumination.

\section{Results}

The best wavelength to differentiate cholesteatoma from bone has been identified as $\lambda=420 \mathrm{~nm}$ for our multispectral 3Dendoscope. The spectral information is augmented using an overlay technique in the broadband mode. The combination with stereoscopic 3D-reconstruction results in new valuable visualization of intraoperative data. The combination enables us to a 3D-model of the patient's anatomy highlighting the identified malicious tissue and compare the sizes with preoperative CT data.

\section{Conclusion}

First results are promising as cholesteatoma can correctly identified, easily highlighted, and visualized as a true-scale 3D model showing the patient specific anatomy. 


\title{
Semi-automatic decision-making process in histopathological specimens from Barrett's carcinoma patients by Hyperspectral imaging (HSI)
}

\author{
Marianne Maktabi, Innovation Center Computer Assisted Surgery (ICCAS) University Leipzig, Leipzig Germany, Ma- \\ rianne.Maktabi@medizin.uni-leipzig.de \\ Yannis Wichmann, Department of Visceral, Transplant, Thoracic and Vascular Surgery, University Hospital of Leipzig, \\ Yannis.Wichmann@medizin.uni-leipzig.de \\ Hannes Köhler, Innovation Center Computer Assisted Surgery (ICCAS) University Leipzig, Leipzig Germany, Han- \\ nes.Koehler2@medizin.uni-leipzig.de \\ Claire Chalopin, Innovation Center Computer Assisted Surgery (ICCAS) University Leipzig, Leipzig Germany, \\ Claire.Chalopin@medizin.uni-leipzig.de \\ Thomas Neumuth, Innovation Center Computer Assisted Surgery (ICCAS) University Leipzig, Leipzig Germany, \\ Thomas.Neumuth@medizin.uni-leipzig.de \\ Andreas Melzer, Innovation Center Computer Assisted Surgery (ICCAS) University Leipzig, Leipzig Germany, An- \\ dreas.Melzer@medizin.uni-leipzig.de \\ Boris Jansen-Winkeln, Department of Visceral, Transplant, Thoracic and Vascular Surgery, University Hospital of Leip- \\ zig, Boris.Jansen-Winkeln@medizin.uni-leipzig.de \\ Henning Ahle, Department of General and Visceral Surgery, Sana Clinic Offenbach GmbH, Offenbach, Germany, Hen- \\ ning.Ahle@Sana.de \\ Dietmar Lorenz, Department of General, Visceral and Thoracic Surgery, Municipal Hospital of Darmstadt GmbH, Darm- \\ stadt, Germany, dietmar.lorenz@mail.klinikum-darmstadt.de \\ Michael Bange, Institute of Pathology, Sana Clinic Offenbach GmbH, Offenbach, Germany, michael.bange@ sana.de \\ Susanne Braun, Institute of Pathology, Sana Clinic Offenbach GmbH, Offenbach, Germany, susanne.braun@sana.de \\ Ines Gockel, Department of Visceral, Transplant, Thoracic and Vascular Surgery, University Hospital of Leipzig, \\ Ines.Gockel@medizin.uni-leipzig.de \\ René Thieme, Department of Visceral, Transplant, Thoracic and Vascular Surgery, University Hospital of Leipzig, \\ Rene.Thieme@medizin.uni-leipzig.de
}

\section{Introduction}

Discrimination of malignant and non-malignant cells of histopathologic specimens is a key step in cancer diagnosics. eminent. Hyperspectral imaging (HSI), as recently applied in medicine, is a novel technology combining imaging with spectroscopy. HSI allows the determination of a spectrum between the visual and near-infrared light (500-1000nm) and it might by the usage used to identify and classify cancer cells by deep lerning alirithms.

\section{Methods (14 pt bold)}

After surgical resection, specimens ( $\mathrm{n}=56)$ of Barrett's cancer tumor samples were fixed in $4 \%$ formaldehyde and sliced $3 \mu \mathrm{m})$. The slides were stained with hematoxylin and eosin (HE) in a standardized manner. Afterwards, they were recorded with a microscopy HSI camera system. Four different classes were annotated and evaluated in a cross-validation by using several machine learning algorithms.

\section{Results (14 pt bold)}

The SVM with a poly kernel showed the worst results. The MLP had the best results (90\% sensitivity and F1-Score) for all classes. The sensitivity values of the separate classes EAC and background were up to $25 \%$ higher than for stroma and squamous epithelium.

\section{Conclusion (14 pt bold)}

For the first time in literature, we were able to analyze esophageal adenocarcinoma, tumor stroma and squamous epithelium cells using HSI. Neuronal network with an MLP algorithm provided the best performance. 


\section{Detecting Bacteria on Wounds with Hyperspectral Imaging in Fluo- rescence Mode}

Bert. H. Herrmann Hochschule Wismar, University of Applied Sciences, Technology, Business and Design, Faculty of Engineering, Wismar, Germany

Georg Daeschlein, Sebastian von Podewils, Claudia Sicher, Department of Dermatology of the University Medicine Greifswald, Greifswald, Germany

Jana Kuhn, Clinic for Diabetes and Metabolic Diseases, Karlsburg Hospital Dr. Guth GmbH \& Co KG, Karlsburg, Germany; Leibniz Institute for Plasma Science and Technology, Greifswald, Germany.

Kai Masur, Mareike Meister, Leibniz Institute for Plasma Science and Technology, Greifswald, Germany.

Philip Wahl, Diaspective Vision GmbH, Pepelow, Germany.

*Corresponding author: Christoph. Hornberger: Hochschule Wismar, University of Applied Sciences, Technology, Business and Design, Faculty of Engineering, Philipp-Müller-Str. 14, 23966 Wismar, Germany, e-mail: Christoph.Hornberger@hs-wismar.de

\section{Introduction}

Chronic non-healing wounds represent an increasing problem. Oxygen supply, bacteria colonization and other parameters influence the healing process. So far, these parameters cannot be monitored in an objective and routinely manner. Existing methods like the microbiological analysis of wound swabs, mean a great deal of effort and partly a long delay. It will be investigated whether a hyperspectral fluorescence imaging system can support the detection of bacteria.

\section{Methods}

Wound data were collected in a bi-centric, non-randomized, controlled cohort study at the University of Greifswald, Clinic and Polyclinic for Skin Diseases and the Competence Center Diabetes Karlsburg with a hyperspectral fluorescence imaging system (TIVITA ${ }^{\circledR}$ ) using a $405 \mathrm{~nm}$ peak wavelength LED illumination. Beside the fluorescence image recording, a microbiological sampling took place to investigate the bacterial load. So far 42 fluorescence images from 42 patients with diabetic foot ulcer were recorded and analysed with principal component analysis after data pre-processing. After dividing the data into a training and a test dataset it was investigated whether the algorithm can detect bacteria in the wound area.

\section{Results}

After the comparison of the microbiological diagnosis of the smear tests and the bacteria detection of the algorithm a sensitivity $>80 \%$ and a specificity of $100 \%$ is calculated for the HSI algorithm.

As there are only few cubes with a negative result of the microbiological diagnosis the specificity is not significant.

\section{Conclusion}

Hyperspectral imaging in fluorescence mode with an excitation wavelength of $405 \mathrm{~nm}$ seems to be a promising tool for Bacteria detection. A more detailed analysis of the PCA loadings suggest that in future a differentiation between different bacteria could be possible - e.g. a first differentiation between gram positive and gram negative bacteria. So far, there are not enough data for a well-founded statement. 


\section{Simulating a Ground Truth for Transit Time Analysis of Indicator Di- lution Curves}

Michael Reiß, Institute of Biomedical Engineering, Karlsruhe Institute of Technology, Karlsruhe, Germany, publications@ibt.kit.edu

Ady Naber, Institute of Biomedical Engineering, Karlsruhe Institute of Technology, Karlsruhe, Germany

Werner Nahm, Institute of Biomedical Engineering, Karlsruhe Institute of Technology, Karlsruhe, Germany

\section{Introduction}

Transit times of a bolus through an organ can provide valuable information for researchers, technicians and clinicians. Therefore, an indicator is injected and the temporal propagation is monitored at two distinct locations. The transit time extracted from two indicator dilution curves can be used to calculate for example blood flow and thus provide the surgeon with important diagnostic information. However, the performance of methods to determine the transit times $\Delta \mathrm{t}$ cannot be assessed quantitatively due to the lack of a sufficient and trustworthy ground truth derived from in-vivo measurements. Therefore, we propose a method to obtain an in silico generated dataset of indicator dilution curves with a ground truth of the transit time. This method allows variations on shape, sampling rate and noise while being accurate and easily configurable.

\section{Methods}

COMSOL Multiphysics is used to simulate a laminar flow through a pipe containing blood analogue. The dye is modelled as a rectangular function of concentration in a segment of the pipe. Afterwards, a flow is applied and the rectangular function will be diluted. Shape varying dilution curves are obtained by discrete-time measurement of the average dye concentration over different cross-sectional areas of the pipe. One dataset is obtained by duplicating one curve followed by subsampling, delaying and applying noise.

\section{Results}

Multiple indicator dilution curves were simulated, which are qualitatively matching in-vivo measurements. The curves temporal resolution, delay and noise level can be chosen according to the requirements of the field of research.

\section{Discussion and Outlook}

Various datasets, each containing two corresponding dilution curves with an existing ground truth transit time, are now available. With additional knowledge or assumptions regarding the detection-specific transfer function, realistic signal characteristics can be simulated. The accuracy of methods for the assessment of $\Delta \mathrm{t}$ can now be quantitatively compared and their sensitivity to noise evaluated. 


\section{Neurophotonic Scanning System - Towards Automatic Infrared Neu- rostimulation}

Celine Wegner, inomed Medizintechnik GmbH, Emmendingen, Germany, c.wegner@inomed.com

Paul Schlett, Section for Neuroelectronic Systems, Uniklinik Freiburg, Freiburg, Germany, paul.schlett@uniklinikfreiburg.de

Julian Höth, Fraunhofer-Institut für Mikrotechnik und Mikrosysteme IMM, Mainz, Germany, julian.hoeth@imm.fraunhofer.de

Thomas Buckert, ARGES GmbH, Wackersdorf, Germany, thomas.buckert@novanta.com

Thomas Klotzbücher, Fraunhofer-Institut für Mikrotechnik und Mikrosysteme IMM, Mainz, Germany, thomas.klotzbuecher@imm.fraunhofer.de

Ulrich Hofmann, Section for Neuroelectronic Systems, Uniklinik Freiburg, Freiburg, Germany, ulrich.hofmann@klinikum.uni-freiburg.de

Thilo Krüger, inomed Medizintechnik GmbH, Emmendingen, Germany, t.krueger@inomed.com

\section{Introduction}

Intraoperative neuromonitoring is without doubt important for all surgical interventions, where nerve structures are at risk. Mapping techniques for the identification of motor nerves and cortex are available. They rely on manual identification using an electrical stimulation probe. A landmark-based visualization of the results is currently not possible. To overcome these limitations, we are developing a system for automatic stimulation and display of functional tissue.

\section{Methods}

Infrared neurostimulation (INS) was proposed to be a feasible alternative to electrical stimulation of nerves. It provides contactless and artifact-free activation of nerves. For our preclinical experiments we used an infrared diode laser system with a wavelength of $1470 \mathrm{~nm}$. For automatic screening, we developed a scanning system and suitable scan-algorithms in order to provide optimal scanning parameters. Recording of compound muscle action potentials (CMAP) was performed with a differential amplifier and appropriate neurophysiologic software.

\section{Results}

Subunits of the system were combined via multiple interfaces. A control-software merges all relevant functions of the individual parts and parallel use. Marking of tissue was realized with a red pilot laser deflected by the same scanning system.

\section{Conclusion}

With this work we could show, that a system for automatic laser deflection with parallel neurophysiologic recording and subsequent highlighting of irradiated tissue is possible. This system can serve as a tool for further systematic investigations in the field of INS. 


\section{Measurement of the retinal irradiation exposure during diaphano- scopic illumination}

Nicole Sieber, Institute of Medical Engineering and Mechatronics, Ulm University of Applied Sciences, Ulm, Germany, Nicole.Sieber@thu.de

Philipp Kölbl, Institute of Medical Engineering and Mechatronics, Ulm University of Applied Sciences, Ulm, Germany, philippkoelbl@gmx.de

Christian Lingenfelder, Pharmpur GmbH, Koenigsbrunn, Germany, christian-lingenfelder@pharmpur.de

Kathrin Stucke-Straub, Department of Mathematics, Natural and Economic Sciences, Ulm University of Applied Sciences, Ulm, Germany, kathrin.stucke-straub@thu.de

Sebsatian Kupferschmid, Clinic of Ophthalmology, Bundeswehrkrankenhaus Ulm, Germany, LASIK-Ulm@gmx.de Martin Hessling, Institute of Medical Engineering and Mechatronics, Ulm University of Applied Sciences, Ulm, Germany, Martin.Hessling@thu.de

\section{Introduction}

In vitreoretinal surgery, the imaging of the ocular fundus is of great importance. Thus, sufficient bright illumination of the intraocular space is mandatory. However, excessive irradiance can cause irreversible photochemical and thermal damage to the retina. In diaphanoscopic illumination, the retina is particularly at risk due to its proximity to the light source. Therefore, the photochemical and thermal retinal hazards for different applied pressures is determined below.

\section{Methods}

Due to the similarity in physiology and anatomy, porcine eyes can be used as a suitable alternative for human eyes. Therefore, the intraocular brightness is investigated in porcine eyes for different applied pressures $(15,65 \mathrm{and} 115 \mathrm{kPa})$. This is done by inserting a fiber into the eye, which detects light coming from the opposite side of the diapanoscopic illuminated eye.

\section{Results}

With increasing applied pressure of the diaphanoscope on the eye the intraocular irradiance increases. Therefore, the thermal and photochemical hazard also increases, which leads to a reduction of the maximum exposure time. Besides, the intraocular irradiance also depends on the pigmentation of the eye.

\section{Conclusion}

Diaphanoscopic illumination can pose relevant stress on the retina as certain irradiation times are exceeded. In addition, the influence of the pigmentation of the eye on the irradiation can also be expected for humans. 